\section{Oral isotretinoin: ensuring safe use while not limiting access to those who need it}

In a Canadian study published in CMAJ, the finding of 3.1 pregnancies per 1000 female isotretinoin users between 1996-2011 is cause for concern for all prescribers of this medication. ${ }^{1}$ The authors proffer potential reasons for inadvertent fetal exposure to oral isotretinoin, including marketing of generic versions that may have fewer consumer educational resources than the original, online availability without prescription or appropriate risk information, and increased use for milder forms of acne. ${ }^{1}$ The latter requires response from a dermatologic perspective.

The designation of clinical severity is problematic. Acne severity is multidimensional, comprising primary acne lesions (type, lesion intensity, extent and distribution), scarring and psychosocial impact. However, acne guidelines typically refer to clinician assessments based on a static evaluation of primary acne lesions. ${ }^{2}$ Furthermore, acne is a chronic condition with a potential for adverse psychosocial consequences - an impact not directly correlated with clinical severity as judged by clinicians. ${ }^{3}$ Psychosocial impact may be severe despite acne being mild or moderate on clinical examination, while severe acne increases the risk of attempted suicide. In patients requiring oral isotretinoin for severe acne, increased suicide risk was observed even before institution of this medication. ${ }^{4}$

Furthermore, post-acne scarring is not included as a dimension in current acne severity scales. Scarring has been observed in those clinically assessed with mild-tomoderate as well as severe acne. This reflects fluctuation in severity and intense inflammation leading to dermal damage. Post-acne scarring is a marker for more severe acne with its own psychosocial effects and treatment consequences, typically invasive and costly.

Although oral isotretinoin has been the standard of care for severe acne, ${ }^{2}$ the management of moderate acne has evolved with increasing recognition of the public health consequences of prolonged antibiotic use.

Current guidelines recommend limiting the use of oral antibiotics to three to four months to mitigate this risk. ${ }^{5}$ These agents are unlikely to lead to remission and their discontinuation generally leads to acne recurrence, and consequently, the need for an alternative systemic option such as oral isotretinoin. The latter remains the sole agent with the potential for inducing remission in acne. ${ }^{6}$

Pregnancy prevention programs have developed in parallel with the availability of oral isotretinoin in the United States and Canada over the past three decades. In the US, these have evolved through warnings on labels; addition of red label stickers to pharmacies; addition of "avoid pregnancy" icons; patient consent forms; pregnancy testing before start of treatment; selection of two forms of birth control (Pregnancy Prevention Program [PPP], 1988); two pregnancy tests before starting treatment and pharmacist provision of a medication guide with prescriptions; dispensing limited to 30-day supply with no refills (SMART, 2001); monthly pregnancy tests; a registration database for patients, prescribers, pharmacists and wholesalers; qualifying questions for patients and monthly identification of contraceptive methods by patients and doctors (iPLEDGE, 2006). ${ }^{7}$

Despite these increasingly stringent pregnancy risk-management approaches, no significant reduction in pregnancies has been shown with $\mathrm{SMART}^{8}$ or iPLEDGE. ${ }^{9} \mathrm{~A}$ consequence of each, however, has been a sequential reduction in the number of isotretinoin treatment courses and the number of patients treated, with females being disproportionately affected. In Canada, the current program is similar to PPP, but with the addition of two pregnancy tests before starting treatment and monthly pregnancy tests, including one month after discontinuation.

The conclusion stated by the authors, that medical practitioners and female patients must be reminded of fetal risks of this medication - including adherence to pregnancy prevention program mea- sures $^{1}$ - is well founded and timely. Additional considerations are the multidimensional nature of acne severity and the potential for morbidity and mortality in a subgroup of patients. The ongoing challenge with oral isotretinoin in Canada is safe and appropriate use while not limiting access to those who need it.

\section{Jerry K.L. Tan MD}

Dermatologist, Faculty of Medicine, Western University, London, Ont.

\section{Neil Shear MD}

Professor, University of Toronto, Sunnybrook Health Sciences Centre, Toronto, Ont.

- Cite as: CMAJ 2017 April 3;189:E510. doi: $10.1503 / \mathrm{cmaj} .732920$

\section{References}

1. Henry D, Dormuth C, Winquist B, et al.; CNODES (Canadian Network for Observational Drug Effect Studies) Investigators. Occurrence of pregnancy and pregnancy outcomes during isotretinoin therapy. CMAJ 2016;188:723-30.

2. Asai $\mathrm{Y}$, Baibergenova A, Dutil M, et al. Management of acne: Canadian clinical practice guideline. CMAJ 2016;188:118-26.

3. Tan JK, Li Y, Fung K, et al. Divergence of demographic factors associated with clinical severity compared with quality of life impact in acne. $J$ Cutan Med Surg 2008;12:235-42.

4. Sundström A, Alfredsson L, Sjölin-Forsberg G, et al. Association of suicide attempts with acne and treatment with isotretinoin: retrospective Swedish cohort study. BMJ 2010;341:c5812.

5. Zaenglein AL, Pathy AL, Schlosser BJ, et al. Guidelines of care for the management of acne vulgaris. J Am Acad Dermatol 2016;74:945-73.e33.

6. Blasiak RC, Stamey CR, Burkhart CN, et al. Highdose isotretinoin treatment and the rate of retrial, relapse, and adverse effects in patients with acne vulgaris. JAMA Dermatol 2013;149:1392-8.

7. Abroms L, Maibach E, Lyon-Daniel K, et al. What is the best approach to reducing birth defects associated with isotretinoin? PLoS Med 2006; 3:e483.

8. Brinker A, Kornegay C, Nourjah P. Trends in adherence to a revised risk management program designed to decrease or eliminate isotretinoinexposed pregnancies: evaluation of the accutane SMART program. Arch Dermatol 2005;141:563-9.

9. Shin J, Cheetham TC, Wong L, et al. The impact of the iPLEDGE program on isotretinoin fetal exposure in an integrated health care system. $J$ Am Acad Dermatol 2011;65:1117-25.

Competing interests: Dr. Jerry Tan has been an advisor, consultant and/or speaker for Cipher and Roche. Dr. Neil Shear has been an advisor, consultant and/or speaker for Cipher. 